\title{
A COHOMOLOGY THEORY FOR COMMUTATIVE ALGEBRAS. $\mathrm{I}^{1}$
}

\author{
MICHAEL BARR
}

1. Introduction. In the first paper of this series [1], henceforth referred to as $I$, we define a cohomology theory for a commutative algebra $R$ with coefficients in an $R$-module $M$ for which $H^{2}(R, M)$ is the group of singular extensions of $R$ by $M$. In this paper we generalize to a cohomology $H(S, \phi, M)$ where $(S, \phi)$ is a singular extension of $R$ and $M$ is an $R$-module. The second group is again a group of extensions and when $(S, \phi)=\left(R, 1_{R}\right), H(S, \phi, M)=H(R, M)$ as defined in I. This generalization leads to a surprising result: a connected sequence in the first variable.

2. Definitions. Let $K$ be a commutative ring with identity. All rings considered will be unitary $K$-algebras and all modules will also be unitary $K$-modules. Let $R$ be an algebra and $M$ an $R$-module. In I we defined a singular extension $0 \rightarrow M \rightarrow S \rightarrow \phi R \rightarrow 0$ of $R$ by $M$ and morphism of two such extensions. This extension will also be denoted by $(S, \phi)$ or just $S$. If $\Phi:\left(S^{\prime}, \phi^{\prime}\right) \rightarrow(S, \phi)$ is a morphism we will also use $\Phi: S^{\prime} \rightarrow S$ to denote the implied algebra morphism. It will be called a surjection or an extension if the implied morphism is a surjection. In any case, $\operatorname{ker} \Phi, \operatorname{ker} \phi$, and $\operatorname{ker} \phi^{\prime}$ are all $R$-modules. We let $\mathfrak{C}_{R}$ or just $\mathfrak{e}$ denote the category of extensions of $R$ and morphisms of extensions. Then an extension of $(S, \phi)$ is an exact sequence $0 \rightarrow M$ $\rightarrow\left(S^{\prime}, \phi^{\prime}\right) \rightarrow^{\Phi}(S, \phi) \rightarrow 0$ where $\Phi$ is a surjection and $M$ is its kernel. If $M$ and $(S, \phi)$ are held fixed, the notions of equivalence and Baer composition make sense as in I. Then the equivalence classes can be shown to form a group which is denoted by $H^{2}(S, \phi, M)$. We denote by $H^{1}(S, \phi, M)$ the group of derivations of $S$ to $M$ (made into an $S$-module by $\phi$ ).

We define an $n$-long exact sequence over $\mathfrak{C}$ to be an exact sequence

$$
0 \rightarrow M \rightarrow M_{n-2} \rightarrow M_{n-3} \rightarrow \cdots \rightarrow M_{1} \rightarrow\left(S^{\prime}, \phi^{\prime}\right) \stackrel{\Phi}{\rightarrow}(S, \phi) \rightarrow 0
$$

where $0 \rightarrow \operatorname{ker} \Phi \rightarrow\left(S^{\prime}, \phi^{\prime}\right) \rightarrow(S, \phi) \rightarrow 0$ is an extension and $0 \rightarrow M \rightarrow M_{n-2}$ $\rightarrow \cdots \rightarrow M_{1} \rightarrow \operatorname{ker} \Phi \rightarrow 0$ is an ordinary exact sequence of $R$-modules. An $\omega$-long sequence is one like that which has no left end. Again we define equivalence, Baer composition and morphism of long exact

Received by the editors October 24, 1964.

${ }^{1}$ This work has been partially supported by the National Science Foundation under Grant NSF GP-1904. 
sequences. An extension will also be called a short exact sequence. The classes of $n$-long sequences beginning with $M$, ending with $(S, \phi)$ can also be shown to form a group, denoted by $H^{n}(S, \phi, M)$.

Just as in I, a long (resp. short) exact sequence is called generic if it admits a morphism to all others with the same right end (and with the identity map on the right). This will also be called a generic resolution (resp. generic extension).

3. Main results. In this section we indicate how to compute the cohomology just defined as the cohomology of a complex, thus proving, among other things, that it is a group. Two computational theorems are also proved here.

Lemma 1. Suppose $(S, \phi)$ is in $\mathrm{e}$ with $\operatorname{ker} \phi=M$ and $(F, \sigma)$ is a generic extension of $R$. Let $X_{0}$ be an $R$-projective mapping onto $M$ and $G$ be the inessential extension of $F$ by $X_{0}$. Then there is $a \tau: G \rightarrow R$ and $\theta:(G, \tau) \rightarrow(S, \phi)$ which are in $\mathcal{C}$ and are a generic extension of $(S, \phi)$.

Proof. $\tau$ is just the composition $G \rightarrow F \rightarrow R$. $\theta$ is the sum of $X_{0} \rightarrow M$ $\rightarrow S$ and any map of $G$ to $S$ which commutes with $\phi$. These are easily seen to be in $\mathcal{C}$. We now must show that $\theta:(G, \tau) \rightarrow(S, \phi)$ is generic. So suppose $\Phi:\left(S^{\prime}, \phi^{\prime}\right) \rightarrow(S, \phi)$ is in $\mathcal{C}$. We have the following commutative diagram:

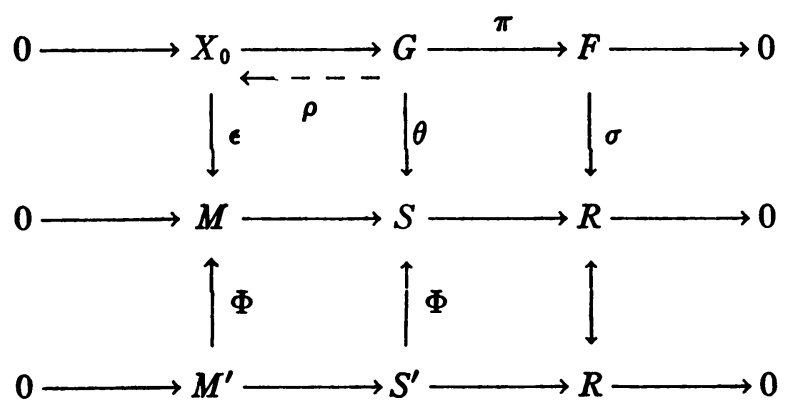

where $\rho: G \rightarrow X_{0}$ is the projection. We begin by choosing $\alpha: F \rightarrow S^{\prime}$ with $\phi^{\prime} \alpha=\sigma$. Then $\phi \Phi \alpha \pi=\phi^{\prime} \alpha \pi=\sigma \pi=\phi \theta$ so that $\phi(\Phi \alpha \pi-\theta)=0$. This means that $\Phi \alpha \pi-\theta$ maps $G$ to $M$ and its restriction to $X_{0}$ is easily seen to be an $R$-morphism. Since $X_{0}$ is projective we can find $\beta: X_{0}$ $\rightarrow M^{\prime}$ so that $\Phi \beta=\Phi \alpha \pi-\theta$ on $X_{0}$. Then restricted to $X_{0}, \theta=\Phi(\alpha \pi-\beta \rho)$. Thus $\theta$ and $\Phi(\alpha \pi-\beta \rho)$ agree on $X_{0}$ and agree when followed by $\phi$. Thus their difference induces a map $\gamma: F \rightarrow M$ which is easily seen to be a derivation. But since $F$ is generic this means that we can find a derivation $\delta: F \rightarrow M^{\prime}$ with $\Phi \delta=\gamma$. Then $\Phi \delta \pi=\Phi(\alpha \pi-\beta \rho)-\theta$ or $\theta=\Phi(\alpha \pi-\beta \rho-\delta \pi)$ and so $\theta^{\prime}=\alpha \pi-\beta \rho-\delta \pi$ is the desired map. The 
reader should check that it is an algebra homomorphism. Recall in doing so that $M^{\prime 2}=0$.

LEMmA 2. Let $0 \rightarrow M \rightarrow G \rightarrow S \rightarrow 0$ a generic extension of $S$ and $X \rightarrow M^{\circ}$ $\rightarrow 0$ an $R$-projective resolution of $M$, then $X \rightarrow G \rightarrow S \rightarrow 0$ is a generic resolution of $S$.

Proof. This is proved exactly as in I.

Theorem 3. Generic resolutions exist.

Proof. This follows from the existence of generic resolutions of $R$, shown in I, together with Lemmas 1 and 2 .

Theorem 4. Let $X \rightarrow G \rightarrow S \rightarrow 0$ a generic resolution of $S$, then $H(S, \phi, M)$ is just the homology of

$$
0 \rightarrow \operatorname{Der}(G, M) \rightarrow \operatorname{Hom}_{R}(X, M) \text {. }
$$

Proof. The proof is left to the reader. It is a standard computation in homological algebra.

TheOREM 5. Let $0 \rightarrow M \rightarrow S^{\prime} \rightarrow S \rightarrow 0$ be a short exact sequence, then we have connecting morphisms $\operatorname{Ext}_{R}^{t-1}(M, N) \rightarrow H^{i+1}(S, \phi, N)$ such that the following sequence is exact,

$$
\begin{aligned}
0 & \rightarrow \operatorname{Der}(S, N) \rightarrow \operatorname{Der}\left(S^{\prime}, N\right) \rightarrow \operatorname{Hom}_{R}(M, N) \rightarrow B^{2}(S, \phi, N) \\
& \rightarrow H^{2}\left(S^{\prime}, \phi^{\prime}, N\right) \rightarrow \cdots \cdots \rightarrow B^{i}(S, \phi, N) \rightarrow B^{i}\left(S^{\prime}, \phi^{\prime}, N\right) \\
& \rightarrow \operatorname{Ext}_{R}^{i-1}(M, N) \rightarrow B^{i+1}(S, \phi, N) \rightarrow \cdots
\end{aligned}
$$

Proof. Let $X \rightarrow M \rightarrow 0$ be an $R$-projective resolution of $M$ and $Y \rightarrow G \rightarrow S \rightarrow 0$ be a generic resolution of $S$. Then we have

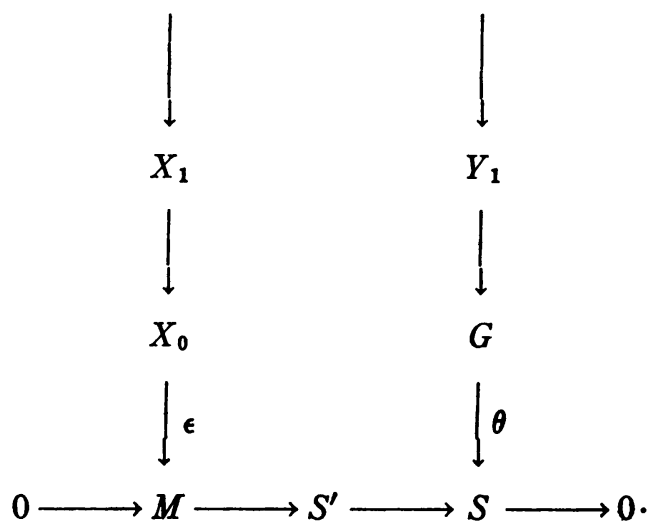

Now let $G^{\prime}$ be the inessential extension of $G$ by $X_{0}$. Then $G^{\prime}$ is a generic resolution of $S^{\prime}$, exactly as was proved in Lemma 1 . Let $L$ be 
the kernel of this extension. We have $0 \rightarrow \operatorname{ker} \epsilon \rightarrow L \rightarrow \operatorname{ker} \theta \rightarrow 0$ is exact as is well known (or see Lemma 6 below). We can let $Z_{i}=X_{i} \oplus Y_{i}$ and $\ldots \rightarrow Z_{i} \rightarrow \cdots \rightarrow Z_{2} \rightarrow Z_{1} \rightarrow L \rightarrow 0$ will be a projective resolution of $L$. The remainder is a standard computation.

Before proceeding further, we need three lemmas.

LEMMA 6. Suppose we have a commutative diagram

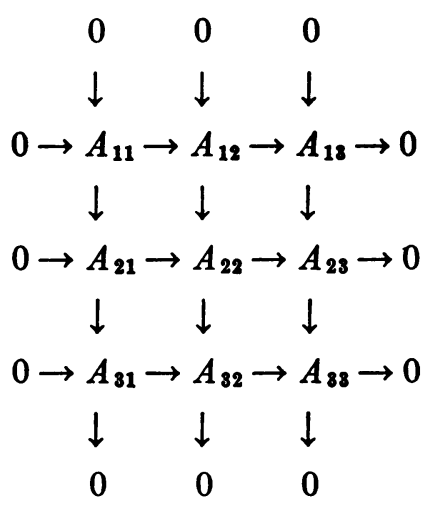

in which all three columns, the middle row and one other row are exact, then the remaining row is exact.

Proof. This is easily proved by diagram chasing.

LEMMA 7. Suppose we have a commutative diagram with exact rows and columns,

$$
\begin{aligned}
& 0 \\
& \downarrow \\
& A_{11} \rightarrow A_{12} \rightarrow A_{18} \rightarrow 0 \\
& \downarrow \downarrow \downarrow \downarrow \\
& A_{21} \rightarrow A_{22} \rightarrow A_{28} \rightarrow 0 \\
& \downarrow \downarrow \downarrow \downarrow \\
& 0 \rightarrow A_{31} \rightarrow A_{32} \rightarrow A_{33} \rightarrow 0 \\
& \downarrow \downarrow \downarrow \downarrow \\
& 0 \quad 0 \quad 0
\end{aligned}
$$

then there is naturally induced an exact sequence,

$$
0 \rightarrow A_{18} \oplus A_{31} \rightarrow A_{22} / A_{11} \rightarrow A_{38} \rightarrow 0 .
$$

Proof. This can be proved by diagram chasing together with some computation. The key step is showing that 


$$
\operatorname{Im}\left(A_{21} \rightarrow A_{22}\right) \cap \operatorname{Im}\left(A_{12} \rightarrow A_{22}\right)=\operatorname{Im}\left(A_{11} \rightarrow A_{22}\right) .
$$

Lemma 8. Suppose $0 \rightarrow A_{1} \rightarrow A_{2} \rightarrow A_{3} \rightarrow 0$ and $0 \rightarrow B_{1} \rightarrow B_{2} \rightarrow B_{3} \rightarrow 0$ are exact sequences of $R$-modules and $\operatorname{Tor}_{1}^{R}\left(A_{3}, B_{3}\right)=0$, then we have an exact sequence $0 \rightarrow A_{1} \otimes_{R} B_{3} \oplus A_{3} \oplus_{R} B_{1} \rightarrow A_{2} \otimes_{R} B_{2} / A_{1} \otimes_{R} B_{1} \rightarrow$ $A_{8} \otimes_{R} B_{8} \rightarrow 0$.

Proof. This is an easy application of Lemma 7.

In what follows, $\otimes$, Tor, "flat," will mean $\otimes_{K}$, Tor ${ }^{K}$, and " $K$-flat" respectively.

Theorem 9. Suppose that $R^{\prime}$ and $R^{\prime \prime}$ are flat algebras and that $\left(S^{\prime}, \phi^{\prime}\right)$ and $\left(S^{\prime \prime}, \phi^{\prime \prime}\right)$ are extensions of $R^{\prime}$ and $R^{\prime \prime}$ with kernels $M^{\prime}$ and $M^{\prime \prime}$ respectively. Suppose $\operatorname{Tor}_{1}\left(S^{\prime}, S^{\prime \prime}\right)=0$. Then if we let

$$
S=S^{\prime} \otimes S^{\prime \prime} / M^{\prime} \otimes M^{\prime \prime} \text {, and } R=R^{\prime} \otimes R^{\prime \prime}
$$

then $\phi^{\prime} \otimes \phi^{\prime \prime}$ induces a map $\phi: S \rightarrow R$ which is a singular extension and for any $R$-module $M$ we have $H(S, \phi, M) \approx H\left(S^{\prime}, \phi^{\prime}, M\right) \oplus H\left(S^{\prime \prime}, \phi^{\prime \prime}, M\right)$.

Proof. The first assertion is clear and we have from Lemma 8 that ker $\phi=M^{\prime} \otimes R^{\prime \prime} \oplus R^{\prime} \otimes M^{\prime \prime}$. If $0 \rightarrow L^{\prime} \rightarrow F^{\prime} \rightarrow R^{\prime} \rightarrow 0$ and $0 \rightarrow L^{\prime \prime} \rightarrow F^{\prime \prime}$ $\rightarrow R^{\prime \prime} \rightarrow 0$ are generic resolutions then it is shown in $I$ that $0 \rightarrow L^{\prime} \otimes R^{\prime \prime} \oplus R^{\prime} \otimes L^{\prime \prime} \rightarrow F^{\prime} \otimes F^{\prime \prime} / L^{\prime} \otimes L^{\prime \prime} \rightarrow R \rightarrow 0$ is also generic. Let $X^{\prime} \rightarrow M^{\prime} \rightarrow 0$ and $X^{\prime \prime} \rightarrow M^{\prime \prime} \rightarrow 0$ be $R^{\prime}$-projective and $R^{\prime \prime}$-projective resolutions of $M^{\prime}$ and $M^{\prime \prime}$ respectively. Then if $G$ and $G^{\prime}$ are the inessential extensions of $F^{\prime}$ by $X_{0}^{\prime}$ and $F^{\prime \prime}$ by $X_{0}^{\prime \prime}$ respectively, they are generic extensions of $S^{\prime}$ and $S^{\prime \prime}$. Let $N^{\prime}=\operatorname{ker}\left(G^{\prime} \rightarrow S^{\prime}\right)$ and $N^{\prime \prime}$ $=\operatorname{ker}\left(G^{\prime \prime} \rightarrow S^{\prime \prime}\right)$. Then

$\left(^{*}\right) \quad 0 \rightarrow N^{\prime} \otimes S^{\prime \prime} \oplus S^{\prime} \otimes N^{\prime \prime} \rightarrow G^{\prime} \otimes G^{\prime \prime} / N^{\prime} \otimes N^{\prime \prime} \rightarrow S^{\prime} \otimes S^{\prime \prime} \rightarrow 0$

is exact by Lemma 8. Also one can check that $\operatorname{ker}\left(G^{\prime} \rightarrow R^{\prime}\right)$ is $X_{0}^{\prime} \oplus L^{\prime}$ and $\operatorname{ker}\left(G^{\prime \prime} \rightarrow R^{\prime \prime}\right)$ is $X_{0}^{\prime \prime} \oplus L^{\prime \prime}$. Then we have

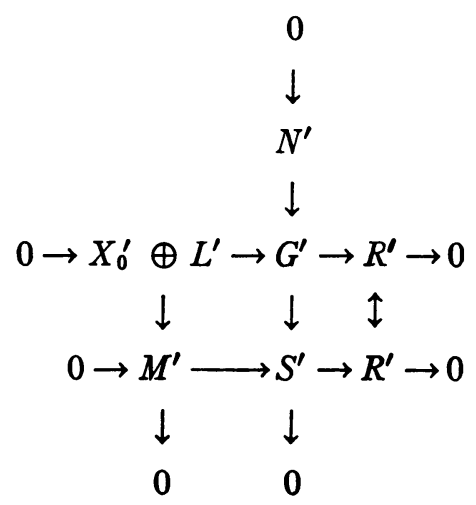


from which, by Lemma $6, N^{\prime}=\operatorname{ker}\left(X_{0}^{\prime} \oplus L^{\prime} \rightarrow M^{\prime}\right)$ and similarly $N^{\prime \prime}=\operatorname{ker}\left(X_{0}^{\prime \prime} \oplus L^{\prime \prime} \rightarrow M^{\prime \prime}\right)$. Now $\operatorname{Tor}_{1}\left(S^{\prime}, S^{\prime \prime}\right)=0$, a fact we have already used. But together with the fact that $R^{\prime}$ and $R^{\prime \prime}$ are flat and the following two exact sequences

$0=\operatorname{Tor}_{2}\left(M^{\prime}, R^{\prime \prime}\right) \rightarrow \operatorname{Tor}_{1}\left(M^{\prime}, M^{\prime \prime}\right) \rightarrow \operatorname{Tor}_{1}\left(M^{\prime}, S^{\prime \prime}\right) \rightarrow \operatorname{Tor}_{1}\left(M^{\prime}, R^{\prime \prime}\right)=0$,

$0=\operatorname{Tor}_{2}\left(R^{\prime}, S^{\prime \prime}\right) \rightarrow \operatorname{Tor}_{1}\left(M^{\prime}, S^{\prime \prime}\right) \rightarrow \operatorname{Tor}_{1}\left(S^{\prime}, S^{\prime \prime}\right) \rightarrow \operatorname{Tor}_{1}\left(R^{\prime}, S^{\prime \prime}\right)=0$

we conclude also that

$$
\operatorname{Tor}_{1}\left(M^{\prime}, M^{\prime \prime}\right)=0 .
$$

But this permits another application of Lemma 8 to have an exact sequence

$$
\begin{aligned}
0 & \rightarrow N^{\prime} \otimes M^{\prime \prime} \oplus M^{\prime} \otimes N^{\prime \prime} \\
& \rightarrow\left(X_{0}^{\prime} \oplus L^{\prime}\right) \otimes\left(X_{0}^{\prime \prime} \oplus L^{\prime \prime}\right) / N^{\prime} \otimes N^{\prime \prime} \rightarrow M^{\prime} \otimes M^{\prime \prime} \rightarrow 0 .
\end{aligned}
$$

Now we may combine $\left(^{*}\right)$ and $\left(^{* *}\right)$ to get a commutative diagram

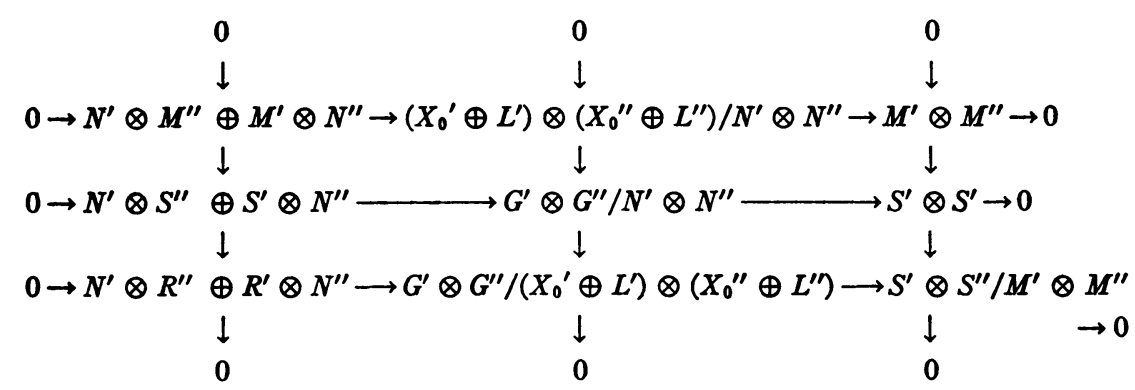

The top two rows have been shown to be exact, the left column is exact because $R^{\prime}$ and $R^{\prime \prime}$ are flat and the other two columns are obviously exact. Hence, by Lemma 6 , the bottom row is also exact. Now we know that $F^{\prime} \otimes F^{\prime \prime} / L^{\prime} \otimes L^{\prime \prime}$ is a generic extension of $R^{\prime} \otimes R^{\prime \prime}$ and that the ker of $\phi$ is $M^{\prime} \otimes R^{\prime \prime} \oplus R^{\prime} \otimes M^{\prime \prime}$. An $R^{\prime} \otimes R^{\prime \prime}$-projective mapping onto this is $X_{0}^{\prime} \otimes R^{\prime \prime} \oplus R^{\prime} \otimes X_{0}^{\prime \prime}$. (See proof of Theorem 7 of I for details.) Then a generic extension of $S$ is the inessential extension of $F^{\prime} \otimes F^{\prime \prime} / L^{\prime} \otimes L^{\prime \prime}$ by $X_{0}^{\prime} \otimes R^{\prime \prime} \oplus R^{\prime} \otimes X_{0}^{\prime \prime}$. We can map $G^{\prime} \otimes G^{\prime \prime} /\left(X_{0}^{\prime} \oplus L^{\prime}\right) \otimes\left(X_{0}^{\prime \prime} \oplus L^{\prime \prime}\right)$ to this by sending $\left(x^{\prime}, f^{\prime}\right) \otimes\left(x^{\prime \prime}, f^{\prime \prime}\right)$ $\rightarrow\left(x^{\prime} \otimes \theta^{\prime \prime}\left(f^{\prime \prime}\right), \theta^{\prime}\left(f^{\prime}\right) \otimes x^{\prime \prime}, f^{\prime} \otimes f^{\prime \prime}\right)$ where $\theta^{\prime}: F^{\prime} \rightarrow R^{\prime}$ and $\theta^{\prime \prime}: F^{\prime \prime} \rightarrow R^{\prime \prime}$ are the generic extensions. This map can be checked to be an algebra homomorphism and the following calculation shows it is an isomorphism, 


$$
\begin{aligned}
& G^{\prime} \otimes G^{\prime \prime} /\left(X_{0}^{\prime} \oplus L^{\prime}\right) \otimes\left(X_{0}^{\prime \prime} \oplus L^{\prime \prime}\right) \\
& \\
& \approx\left(X_{0}^{\prime} \oplus F^{\prime}\right) \otimes\left(X_{0}^{\prime \prime} \oplus F^{\prime \prime}\right) /\left(X_{0}^{\prime}+L^{\prime}\right) \otimes\left(X_{0}^{\prime \prime}+L^{\prime \prime}\right) \\
& \approx \frac{\left(X_{0}^{\prime} \otimes X_{0}^{\prime \prime} \oplus X_{0}^{\prime} \otimes F^{\prime \prime} \oplus F^{\prime} \otimes X_{0}^{\prime \prime} \oplus F^{\prime} \otimes F^{\prime \prime}\right)}{\left(X_{0}^{\prime} \otimes X_{0}^{\prime \prime} \oplus X_{0}^{\prime} \otimes L^{\prime \prime} \oplus L^{\prime} \otimes X_{0}^{\prime \prime} \oplus L^{\prime} \otimes L^{\prime \prime}\right)} \\
& \approx X_{0}^{\prime} \otimes R^{\prime \prime} \oplus R^{\prime} \otimes X_{0}^{\prime \prime} \oplus\left(F^{\prime} \otimes F^{\prime \prime} / L^{\prime} \otimes L^{\prime \prime}\right) .
\end{aligned}
$$

Hence the bottom row of the above diagram is a generic extension of $S$. The remainder follows exactly as in Theorem 7 of $\mathrm{I}$.

TheOREM 10. Let $(S, \phi)$ be an extension of $R$ with kernel $N$. Suppose that $A$ is a multiplicatively closed subset of $R$ not containing 0 and that it does not have any zero divisors of $R$. Let $B=\phi^{-1}(A)$. Then $\phi$ induces a map $\phi_{B}: S_{B} \rightarrow R_{A}$ which is a singular extension and for any $R_{A}$ module $M$ we have $H(S, \phi, M) \approx H\left(S_{B}, \phi_{B}, M\right)$.

Proof. From the proof of Theorem 8 of $I$ we have an exact sequence $0 \rightarrow N \otimes_{R} R_{A} \rightarrow S_{B} \rightarrow \phi_{B} R_{A} \rightarrow 0$ which is a singular extension and defines $\phi_{B}$. Now let $0 \rightarrow L \rightarrow G \rightarrow{ }^{\theta} S \rightarrow 0$ be a generic singular extension of $S$. In exactly the same way we have $0 \rightarrow L \otimes_{S} S_{B} \rightarrow G_{C} \rightarrow S_{B} \rightarrow 0$ where $C=\theta^{-1}(B)$ is exact. (An examination of the proof of Theorem 8 of I will show that it is not required that $B$ contain no zero divisor of S.) Now

$$
L \otimes_{S} N \otimes_{R} R_{A} \rightarrow L \otimes_{S} S_{B} \rightarrow L \otimes_{S} R_{A} \rightarrow 0
$$

is exact. But the image in $S_{B}$ of $N \otimes_{R} R_{A}$ is just $N \cdot S_{B}$ and $L \otimes_{S} N^{\prime} S_{B}$ $=L \cdot N \otimes_{S} S_{B}=0$ since $L$ is an $R$-module. Thus $L \otimes_{S} S_{B} \approx L \otimes_{S} R_{A}$. But since $L$ and $R_{A}$ are $R$-modules, $L \otimes_{S} R_{A} \approx L \otimes_{R} R_{A}$. Hence, $0 \rightarrow L \otimes_{R} R_{A}$ $\rightarrow G_{C} \rightarrow S_{B} \rightarrow 0$ is a generic extension of $S_{B}$. The rest follows exactly as in I, Theorem 8.

4. Remarks. There still remains to consider the relative theory. The problem is this: Given a subring $K^{\prime} \subset K$ describe the cohomology group $H_{K^{\prime}}^{n}(S, \phi, M)$ of all $n$-long sequences which are $K^{\prime}$-split and with two being equivalent if there is an equivalence which is $K^{\prime}$-split. (An arbitrary $f: A \rightarrow B$ is called split if each of the sequences $0 \rightarrow \operatorname{ker} f$ $\rightarrow A \rightarrow$ coim $f \rightarrow 0$ and $0 \rightarrow$ im $f \rightarrow B \rightarrow$ coker $f \rightarrow 0$ is a split exact sequence.) At one extreme we could take $K^{\prime}=K$ and get the $K$-relative theory. At the other extreme we could take $K^{\prime}$ as the subring generated by 1 to get the additively split theory.

\section{BIBLIOGRAPHY}

1. M. Barr, A cohomology theory for commutative algebras. I, Proc. Amer. Math. Soc. 16 (1965), 1379-1384.

UNIVERSITY OF ILLINOIS 